\title{
Structural and Electrical Characterization of Undoped Diamond Layer Grown by HF CVD
}

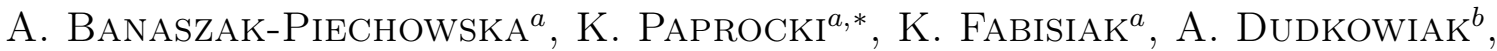 \\ M. SzYBOWICZ ${ }^{b}$ AND E. StARYGA ${ }^{c}$ \\ ${ }^{a}$ Institute of Physics, Kazimierz Wielki University, Powstańców Wielkopolskich 2, 85-090 Bydgoszcz, Poland \\ ${ }^{b}$ Faculty of Technical Physics, PUT, Piotrowo 3, 60-965 Poznań, Poland \\ ${ }^{c}$ Institute of Physics, Technical University of Łódź, Wólczańska 219, 93-005 Łódź, Poland
}

(Received June 20, 2017; in final form July 5, 2017)

\begin{abstract}
The undoped diamond layers were prepared using hot filament chemical vapor deposition technique. The controlled variation of the deposition parameters resulted in the layers with varying amount of nondiamond impurities. Routine characterization of the layers was carried out using scanning electron microscopy, X-ray diffractometry, and the Raman spectroscopy. Detailed measurements of room temperature electrical conductivity $\left(\sigma_{300}\right)$, currentvoltage characteristics have yielded useful information about the electrical conduction mechanism in this interesting material. The $\sigma_{300}$ and $I-V$ characteristic measurements were done in sandwiched configuration taking care off the surface effects. The diamond shows room temperature dc conductivity reaching the values in the range of $\sigma_{300} \approx 0.1-1 \mu \mathrm{S} / \mathrm{cm}$. The $I-V$ characteristics in these layers show space charge limited conduction behavior with $I \sim V^{2}$ in high voltage region. The obtained results are explained in terms of chemically adsorbed hydrogen on the surface of diamond layers, which is a source of acceptor states just above the top of valence band.
\end{abstract}

DOI: 10.12693/APhysPolA.132.1411

PACS/topics: 61.05.cp, 81.05.ug, 73.50.Gr, 73.61.-r

\section{Introduction}

The diamond layers grown by chemical vapor deposition (CVD) have become recently the subject of extensive studies mainly due to their unique combination of thermal, mechanical, and optoelectronic properties. However, the polycrystalline nature of the CVD diamond layers is a bottleneck in the construction of diamond-based electronic devices. Another challenge is the presence of graphitic and nondiamond impurities co-deposited during the growth of diamond layers. On the other hand, the nondiamond impurities, occurring mainly at the grain boundaries, may introduce localized states in the energy gap, which result in the modification of electrical transport mechanism [1-4]. Considering CVD diamond layers as a potential candidate for application in microelectronics it is important to study the electrical transport in the films of different quality, which can be controlled during the CVD growth.

In this work we show results of measurements of the bulk electrical dc conductivity of the polycrystalline diamond layers grown at different deposition pressure. We discuss the results of RT conductivity $\left(\sigma_{300}\right)$ measurements, current-voltage $(I-V)$ characteristics obtained for three representative samples of the CVD diamond layers of different crystalline quality and different concentrations of nondiamond phase.

The variation of $\sigma_{300}$ conductivity and density of states at the Fermi level $N\left(E_{\mathrm{F}}\right)$ are attributed to the chemically bonded $\mathrm{H}$-atoms in the diamond structure.

\footnotetext{
*corresponding author; e-mail: paprocki@ukw.edu.pl
}

\section{Experimental}

The diamond layers have been deposited on the $n$-type Si substrate using home-made HF CVD reactor described elsewhere [5]. The gases flow rates were controlled by calibrated mass flow meters. The tungsten filament temperature was measured by Optris $\mathrm{CT}$ ratio $1 \mathrm{M}$ optical pyrometer while the substrate temperature was controlled by PtRh10-Pt thermocouple. The diamond synthesis was carried out using mixture of methanol (3 vol.\%) and hydrogen (97 vol.\%) at the flow rate of $150 \mathrm{sccm}$ (standard cubic centimeter per min). The all process parameters are summarized in Table I.

The parameters of diamond growth process.

TABLE I

\begin{tabular}{c|c}
\hline \hline Working gas & mixture of methanol and hydrogen \\
methanol concentration & $3.0 \mathrm{vol} . \%$ \\
filament temperature & $2300 \mathrm{~K}$ \\
substrate & $\mathrm{n}$-type Si \\
substrate temperature & $1010 \mathrm{~K}$ \\
gas flow rate & $150 \mathrm{sccm}$ \\
total gas pressure & $20-100 \mathrm{mbar}$
\end{tabular}

The scanning electron microscopy (SEM) microscope type JSM-820 was used to study the morphology of the obtained diamond layer. The Raman spectra were recorded using Renishaw InVia Raman spectrometer equipped with argon laser operating at $488 \mathrm{~nm}$ line using an excitation source. X-ray diffraction spectra were recorded by type Dron-4a X-ray diffractometer, working with a step of 0.05 degree.

\section{Results and discussion}

The SEM photos of morphologies of diamond layer deposited at different pressures are presented in Fig. 1. 


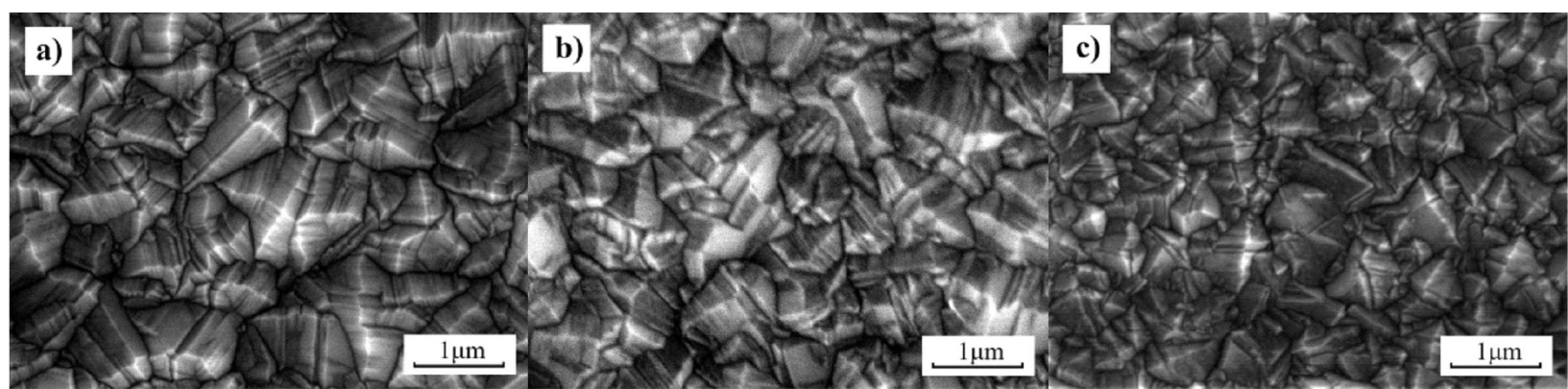

Fig. 1. SEM pictures of diamond layers synthesized at: (a) 20 mbar, (b) 60 mbar, (c) 100 mbar.

The estimated thicknesses of the layers were in the range from 10.2 to $10.6 \mu \mathrm{m}$. As it is seen the obtained morphologies only slightly depend on the working gas pressure. The diamond layer synthesized at $20 \mathrm{mbar}$ is characterized by microcrystalline grains exceeding $1 \mu \mathrm{m}$ in size while the remaining diamond layers are composed of microcrystalline grains smaller than $1 \mu \mathrm{m}$. It means that the working gas pressure has not an essential influence diamond layers morphologies.

Nevertheless, the SEM studies are in agreement with the Raman spectroscopy measurements (Fig. 2 and Table II). The presented Raman spectra of the diamond layers consist of sharp, narrow diamond peak at around $1332 \mathrm{~cm}^{-1}$ and broad $G$-band peaked at 1530 $1560 \mathrm{~cm}^{-1}[6]$.

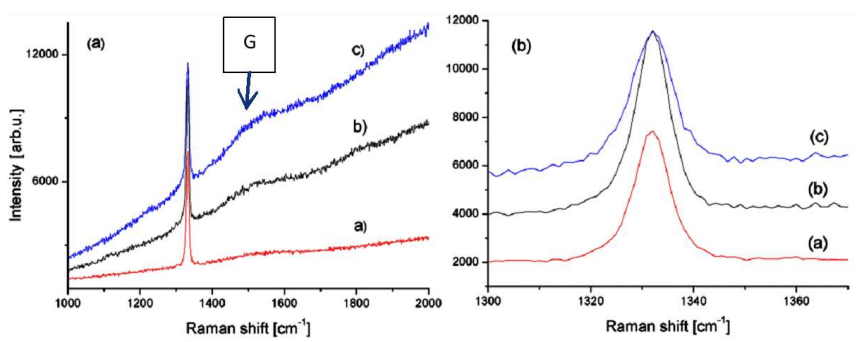

Fig. 2. (a) Raman spectra of diamond films from

Fig. 1, (b) details of diamond Raman line.

The Raman peaks (diamond and $G$-band) are superimposed on a broad luminescence background inclined at different angles. The numerical deconvolution of these spectra, according to the procedure described elsewhere [7] allows to estimate the following parameters of the Raman spectrum: FWHM of diamond line, $s p^{2} / s p^{3}$ ratio and slope $m$ of photoluminescence background. The ratio of $s p^{2} / s p^{3}$ was calculated using formula given by McNamara et al. [7]. The obtained results are collected in Table II.

It is known that broad luminescence background arise from $s p^{2}$-hybridized amorphous carbon admixture in diamond layers and its slope $m$ can be used to determine the hydrogen content. The hydrogen content, associated with $s p^{2}$ carbon phase residing mainly on diamond grain boundaries, can be calculated from the normalized background slope $m$ according to the empirically derived fol- lowing equation [8]:

$$
H[a t . \%]=21.7+16.6 \log \left(m / I_{G}[\mu \mathrm{m}]\right),
$$

where $m$ - slope of PL background of the Raman spectrum, $I_{G}$ - integral intensity of the Raman $G$-band.

The slopes $m$ and hydrogen concentrations in amorphous carbon phase admixture, which is small fraction of diamond layers, are collected in Table II.

TABLE II

Parameters of Raman spectra. Diamond Raman peak position $1331.9\left[\mathrm{~cm}^{-1}\right]$.

\begin{tabular}{l|c|c|c|c|c}
\hline \hline Sample & $\begin{array}{c}\text { Pressure } \\
{[\mathrm{mbar}]}\end{array}$ & $\begin{array}{c}\text { FWHM } \\
{\left[\mathrm{cm}^{-1}\right]}\end{array}$ & $\begin{array}{c}s p^{2} / s p^{3} \\
{[\%]}\end{array}$ & $\begin{array}{c}m \\
{[\mathrm{~cm}]}\end{array}$ & $\begin{array}{c}H \\
{[\%]}\end{array}$ \\
\hline Kf 221 & 20 & 7.2 & 0.30 & 1.9 & 7.5 \\
Kf 230 & 60 & 7.5 & 0.49 & 7.1 & 11.6 \\
Kf 217 & 100 & 8.8 & 0.53 & 10.2 & 21.6
\end{tabular}

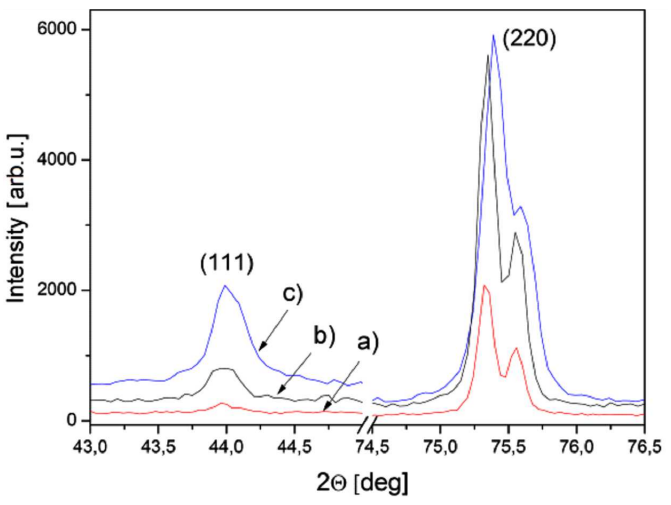

Fig. 3. XRD (111) and (220) peaks of the diamond layers synthesized at different deposition pressures: (a) 20 mbar, (b) 60 mbar, (c) 100 mbar.

Results presented in Table II coincide with SEM images. Diamond films consisting of smaller microcrystalline grains show higher values of both the FWHM and the slope $m$. In order to confirm the observation obtained from the Raman measurements the XRD diffraction was carried out. In Fig. 3 the main (111) and (220) peaks of each samples are presented. Unfortunately, XRD does not prove information on amorphous carbon admixture, which is a main problem in growing high quality diamond 
films, but gives valuable information about diamond layers crystallinity.

The crystalline parameters i.e. the values of lattice spaces $d$ and crystallite sizes $D$ in $\langle 111\rangle$ direction were calculated from XRD spectra and are collected in Table III.

TABLE III

Parameters of XRD spectra $(d-$ interplanar distance, $D$ - crystallite size and $T C_{(h k l)}$ texture factor).

\begin{tabular}{|c|c|c|c|c|c|c|}
\hline \multirow[b]{2}{*}{ Sample } & \multicolumn{3}{|c|}{ (111) } & \multicolumn{3}{|c|}{$(220)$} \\
\hline & $\begin{array}{c}2 \Theta \\
{[\operatorname{deg}]}\end{array}$ & $\begin{array}{c}d \\
{[\AA]}\end{array}$ & $\begin{array}{c}D \\
{[\mathrm{~nm}]}\end{array}$ & $\begin{array}{c}2 \Theta \\
{[\mathrm{deg}]}\end{array}$ & $\begin{array}{c}D \\
{[\mathrm{~nm}]}\end{array}$ & $\begin{array}{c}T C_{(h k l)} \\
{[\%]}\end{array}$ \\
\hline Kf 221 & 43.98 & 2.0587 & 36.09 & 75.34 & 61.11 & 95.1 \\
\hline Kf 230 & 43.97 & 2.0594 & 30.16 & 75.35 & 56.30 & 84.0 \\
\hline Kf 217 & 44.04 & 2.0559 & 28.26 & 75.39 & 40.03 & 64.7 \\
\hline
\end{tabular}

The XRD spectra show a single-phase crystalline diamond structure with noticeable differences in the intensities of the (111), (220) diamond reflexes. As one can see, diamond deposited at 100 mbar is characterized by most intense (220) reflex. Slightly shifted reflexes indicate on internal stress existing in diamond layer. In our case XRD spectra indicate on $\langle 220\rangle$ preferred orientation as it was determined by texture coefficient $T C_{(h k l)}$ defined by the following formula $[9,10]$ :

$$
T C_{(h k l)}=\frac{I_{(h k l)} / I_{0(h k l)}}{(1 / n) \sum_{n} I_{(h k l)} / I_{0(h k l)}},
$$

where $T C_{(h k l)}$ is the texture coefficient of the $(h k l)$ plane, $I_{(h k l)}$ is intensity of the XRD peak corresponding to $(h k l)$ planes, and $n$ is the number of preferred directions of growth (number of the diffraction peaks taken into account). $I_{0(h k l)}$ denotes the intensity of the XRD peak reference of the randomly oriented crystallites and is assumed to be the same as the JCPDS-ICDD (or ASTM) powder diffraction files.

It should be noted that the Raman spectroscopy and XRD measurements clearly indicate on the differences between the samples, although their morphologies are very similar.

From the Raman and XRD measurements one can conclude that the studied diamond layers show noticeable differences in hydrogen concentration (slope $m$ of background of the Raman spectra, $G$ - band intensity) and in crystallites sizes $D$ (XRD). It is well known that "as-grown" CVD polycrystalline diamond films exhibit $p$-type conductivity which can be removed by oxidation [11, 12]. Ballutaud et al. showed that the hydrogen concentration in polycrystalline diamond layer increases with increase of $s p^{2} / s p^{3}$ ratio, and increase with decrease of crystallite sizes [13] which is in agreement with our observations (see Tables II and III). According to Zhang et al. [14] chemically bonded hydrogen should govern the electrical properties of diamond layer. The $I-V$ characteristics recorded for each sample in $\mathrm{Au} / n$-Si/diamond/Au heterostructure configuration exhibit rectifying behavior (Fig. 4a).

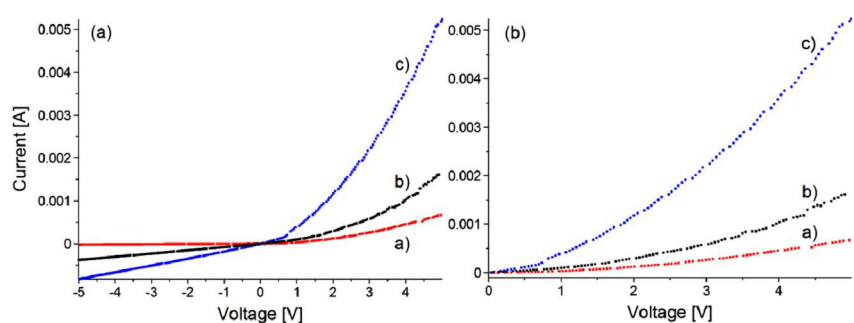

Fig. 4. (a) $I-V$ characteristics recorded at RT, (b) $I-V$ forward characteristics recorded at RT for diamond layers presented in Fig. 1.

As it is seen, each sample exhibits different rectifying properties of which depend on their structural properties.

The analysis of $I-V$ characteristic in forward direction (Fig. 4b) shows two different characters depending on the voltage regions. For the region of low voltages $(<0.7 \mathrm{~V})$ the $I-V$ characteristics show linear dependence of $I$ vs. $V$ as it shown in Fig. 5a. For higher voltages $(>0.7 \mathrm{~V})$ the linear dependence is observed for the plot $I$ vs. $V^{2}$, according to Eq. (3).

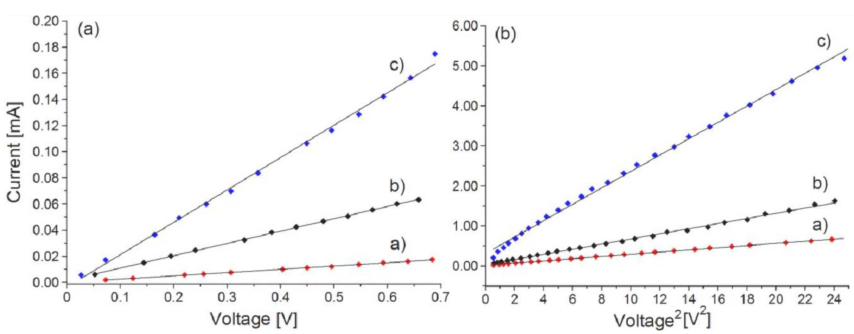

Fig. 5. $\quad I-V$ characteristic, recorded at $\mathrm{RT}$, for regions of: (a) low voltages $(<0.7 \mathrm{~V})$, (b) higher voltages $(>0.7 \mathrm{~V})$ for diamond layers presented in Fig. 1.

For higher voltages $I-V$ forward current is controlled by space charge limited current (SCLC) mechanism and the dependence of $I$ vs. $V$ can be described by the following formula [15]:

$$
I=\frac{9}{8} \varepsilon \varepsilon_{0} \theta \mu_{0} \frac{v^{2}}{d^{3}},
$$

where $\theta$ is the ratio of free to trapped charges at given temperature, $\mu_{0}$ - hole mobility, $d$ - diamond layers thickness, $\varepsilon-$ dielectric constant.

From the slope of the plots shown in Fig. 5a one can estimate the specific electric conductivity using the Ohm law. The estimated values of $\sigma_{300}$ are collected in Table IV.

TABLE IV

Estimated values of dc-conductivity $\sigma_{300}$ and density of states at the Fermi level $N\left(E_{\mathrm{F}}\right)$.

\begin{tabular}{c|c|c}
\hline \hline Sample & $\begin{array}{c}\sigma_{300} \\
{[\mu \mathrm{S} / \mathrm{cm}]}\end{array}$ & $\begin{array}{c}N\left(E_{\mathrm{F}}\right) \\
{\left[\mathrm{nm}^{-3} \mathrm{meV}^{-1}\right]}\end{array}$ \\
\hline Kf 221 & 0.12 & 71 \\
Kf 230 & 0.51 & 81 \\
Kf 217 & 1.26 & 131
\end{tabular}


The region of SCLC can be used to calculate the density of states (DOS) near the Fermi level $N\left(E_{\mathrm{F}}\right)$.

One of the methods which allows for DOS calculation is the method proposed by Den Boer [16]. According to his method the DOS near Fermi level is given by

$$
N\left(E_{\mathrm{F}}\right)=\frac{2 \varepsilon \varepsilon_{0} \Delta V}{e d^{2} \Delta E_{\mathrm{F}}},
$$

where $\varepsilon \varepsilon_{0}$ - permittivity of diamond layer ( $\varepsilon$ is assumed to be 5.5 as for natural diamond), $e$ - electronic charge, $\Delta E_{\mathrm{F}}$ - the shift of the Fermi level due to the change of voltage $\Delta V=V_{2}-V_{1}$ and is given by formula

$$
\Delta E_{\mathrm{F}}=k T \ln \frac{I_{2} V_{1}}{I_{1} V_{2}},
$$

where $I_{1}$ and $I_{2}$ are the currents measured at voltages $V_{1}$ and $V_{2}$, respectively.

The values of estimated density of states $N\left(E_{\mathrm{F}}\right)$ at the Fermi level are collected in Table IV.

The data from Table IV show that both specific conductivity $\sigma_{300}$ and density of states at the Fermi level $N\left(E_{\mathrm{F}}\right)$ increase with increase of hydrogen concentration measured by slope $m$ (Table II) and with decrease of crystallites sizes (Table III).

\section{Conclusions}

Results of SEM, XRD, the Raman scattering spectroscopy, and electrical measurements carried out on the samples of HF CVD diamond layers reveal correlations between HF CVD growth parameters, morphology, crystalline structure and electrical properties of the obtained films. The crystallographic quality and purity deduced from the Raman spectroscopy (FWHM and $s p^{2} / s p^{3}$ ) and XRD (crystallite sizes, preferred orientation) have a direct impact on the electrical properties of diamond films. As-grown polycrystalline CVD has a relatively high near surface concentration of hydrogen, presumably due to the way that CVD growth is typically terminated.

The current study shows that it is hydrogen within diamond that gives rise to carriers; shallow acceptor states and possibly other deeper gap states must form when hydrogen is within the diamond lattice in these concentrations. This is in agreement with previous reports $[17,18]$ which proposed that the carriers arise though formation of shallow acceptor states beneath the surface when hydrogen is present. It was suggested that the chemically bonded hydrogen causes band bending leading to an accumulation of hole in this region $[19,20]$. It means that the H-terminated surfaces are critically important in achieving desired electrical properties.

Our results confirm above suggestions. The room temperature $\sigma_{300}$ conductivity calculated from linear portion of the $I-V$ curves in low voltage region was in the range of $0.1-1 \mu \mathrm{S} / \mathrm{cm}$ and the higher values of $\sigma_{300}$ corresponds to the diamond layer characterized by higher $s p^{2} / s p^{3}$ ratios (Kf 217). The $N\left(E_{\mathrm{F}}\right)$ calculated from $I-V$ characteristics for SCLC region showed higher values (Table IV) for the higher slope $m$ of the luminescence background of the corresponding Raman spectra. Obtained results clearly suggest that acceptor states associated with chemically bonded hydrogen are mainly responsible for charge transport in undoped CVD diamond layers.

The exact nature of the hydrogen promoting shallow acceptor states remains still not fully explained and is worthy of further investigation.

\section{Acknowledgments}

This work was supported in part by Project of Kazimierz Wielki University for development of basic research.

\section{References}

[1] M. Kubovic, M. Kasu, I. Kallfass, M. Neuburger, A. Aleksov, G. Koley, M.G. Spencer, E. Kohn, Diam. Relat. Mater. 13, 802 (2004).

[2] F. Sicignano, A. Vellei, M.C. Rossi, G. Conte, S. Lavanga, C. Lanzieri, A. Cetronio, V. Ralchenko, Diam. Relat. Mater. 16, 1016 (2007).

[3] G. Conte, M.C. Rossi, S. Salvatori, F. Tersigni, P. Ascarelli, E. Cappelli, Appl. Phys. Lett. 82, 4459 (2003).

[4] M.M. Bataineh, D.K. Reinhard, Diam. Relat. Mater. 6, 1689 (1997).

[5] K. Fabisiak, W. Marciniak, S. Orzeszko, F. Rozploch, Diam. Relat. Mater. 1, 83 (1992).

[6] K. Fabisiak, A. Banaszak, M. Kaczmarski, M. Kozanecki, Opt. Mater. 28, 106 (2006).

[7] K.M. McNamara, K.K. Gleason, D.J. Vestyck, J.E. Butler, Diam. Relat. Mater. 1, 1145 (1992).

[8] C. Casiraghi, F. Piazza, A. Ferrari, D. Grambole, J. Robertson, Diam. Relat. Mater. 14, 1098 (2005).

[9] D.I. Rusu, G.G. Rusu, D. Luca, Acta Phys. Pol. A 119, 850 (2011).

[10] C.T. Kuo, C.R. Lin, H.M. Lien, Thin Solid Films 290-291, 254 (1996)

[11] H.J. Looi, L.Y. Pang, A.B. Molloy, F. Jones, M.D. Whitfield, J.S. Foord, R.B. Jackman, Carbon 37, 801 (1999).

[12] A. Kulkarni, A. Shrotriya, P. Cheng, H. Rodrigo, R. Bashyam, D. Keeble, Thin Solid Films 253, 141 (1994).

[13] D. Ballutaud, F. Jomard, T. Kociniewski, E. Rzepka, H. Girard, S. Saada, Diam. Relat. Mater. 17, 451 (2008).

[14] W. Zhang, J. Ristein, L. Ley, Phys. Rev. E 78 041603 (2008).

[15] T. Abdel-Malik, Int. J. Electron. 72, 409 (1992).

[16] W. Den Boer, J. Phys. Colloq. 42, C4 (1981).

[17] K. Hayashi, S. Yamanaka, H. Watanabe, T. Sekiguchi, H. Okushi, K. Kajimura, J. Appl. Phys. 81, 744 (1997).

[18] K. Kimura, K. Nakajima, S. Yamanaka, M. Hasegawa, H. Okushi, Appl. Phys. Lett. 78, 1679 (2001).

[19] J. Shirafuji, T. Sugino, Diam. Relat. Mater. 5, 706 (1996).

[20] H. Kawarada, H. Sasaki, A. Sato, Phys. Rev. B 52, 11351 (1995). 